\title{
Noticias falsas; en busca de la vacuna
}

\author{
Fake news; looking for a vaccinee \\ Edison Caiza $^{1}$ (D), Alejandro Fernandez ${ }^{1}$ (D), Diego Torres ${ }^{1,2}$ (iD \\ ${ }^{1}$ Universidad Nacional de La Plata, La Plata, Argentina \\ ${ }^{2}$ Universidad Nacional de Quilmes, Bernal, Argentina \\ edison.caizaa@lifia.info.unlp.edu.ar, alejandro.fernandez@lifia.info.unlp.edu.ar, \\ diego.torres@lifia.info.unlp.edu.ar
}

(Recibido: 21 Septiembre 2020; aceptado: 5 Noviembre 2020; Publicado en Internet: 1 Diciembre 2020)

\begin{abstract}
Resumen. La difusión de información falsa representa una seria amenaza para los individuos, entidades y sociedades. En respuesta, se ha intensificado el estudio de las "fake news", la forma en que se producen y difunden, y las medidas para evitar o reducir su propagación. Siguiendo el método de revisión sistemática, el presente trabajo pretende dar respuesta, desde distintas miradas y bajo distintas concepciones, a interrogantes como: ¿qué son las noticias falsas?; ¿cómo se caracteriza su ciclo de vida?; y, ¿cómo se determina el impacto de las noticias falsas? Adicionalmente, y debido a nuestro interés particular en el diseño de espacios seguros para la colaboración en línea, nos preguntamos: ¿existe relación entre la propagación o el impacto de las noticias falsas y el diseño de los espacios colaborativos en los que se diseminan?
\end{abstract}

Palabras clave: Desinformación, Espacios colaborativos, Noticias falsas, Post-verdad, Redes sociales, Revisión de literatura.

Abstract. The dissemination of false information represents a severe threat to individuals, entities, and societies. In response, the study of "fake news" has been intensified, how they are produced, disseminated, and the measures to prevent or reduce its spread. Following the systematic review method, the present research tries to answer questions from different perspectives and under different conceptions: What is fake news? Is there a life cycle for fake news? How is the impact of fake news determined? Additionally, and due to our particular interest in designing safe spaces for online collaboration, we ask ourselves: Is there a relationship between disseminating fake news and the design of the collaborative space in which it is disseminated?

Keywords: Collaborative spaces, Disinformation, Fake news, Post-truth, Social media, Literature review.

Tipo de artículo: Artículo de revisión.

\section{Introducción}

Las noticias falsas, como fenómeno contemporáneo, ha generado gran impacto social. Los individuos pueden producir y difundir información inexacta o engañosa de manera sencilla y casi sin restricciones, gracias a las ventajas que ofrecen las plataformas tecnológicas. Esto ha motivado que los académicos de distintas disciplinas aborden la problemática, la estudien y propongan ciertas alternativas para contrarrestarla. Sin embargo, el campo de estudio de las noticias falsas es relativamente nuevo. Gran parte de la literatura científica que se puede consultar se ha producido y publicado apenas hace unos pocos años. Para citar un momento histórico, el auge de las noticias falsas y el dominio en el lenguaje de los ciudadanos y medios especializados, es apenas posterior a las elecciones presidenciales de los Estados Unidos, que ganó Donald Trump en 2016. El incremento de la producción científica coincide con éste evento y con el proceso del Brexit en Reino Unido.

Dado que el fenómeno de las noticias falsas es actual y que su difusión puede causar daño o poner en riesgo a personas, entidades o sociedades, es imprescindible conocer, en primer lugar, sus definiciones y las características notables que las determinan a fin de identificarlas y tratarlas; además de proponer ciertas alternativas para evitar su propagación. En segundo lugar, resulta muy significativo comprender el impacto de producen en las sociedades e individuos que las generan, las difunden y las consumen; así como también precisar la existencia de elementos que permitan evaluar el impacto, de manera cuantitativa o cualitativa. 
Por último, se vuelve crítico identificar las características de los espacios colaborativos que facilitan o impiden la difusión de noticias falsas; solo de esa manera podremos concebirlos (o vacunarlos) para que estos se vuelvan inmunes a ellas. El presente trabajo reporta algunas de las conclusiones a las que llegamos a partir de una meta-revisión sistemática de literatura.

\section{Metodología}

Una búsqueda rápida en Scopus de artículos que mencionen en su título "fake news" (o algún término vinculado como “hoax") arrojó, en Julio de 2020, más de 1500 resultados. En el quinquenio previo al año 2017, el número de artículos es de 20 en promedio; y crece fuertemente a partir de ese año. Los artículos provienen de múltiples disciplinas como ciencias sociales ( $\sim 600$ artículos), ciencias de la computación $(\sim$ 470 artículos), artes y humanidades ( 290 artículos), medicina ( 230 artículos), ingeniería ( $\sim 75$ artículos), y matemáticas ( 119 artículos), entre otras. Cada disciplina intenta, desde su perspectiva, con sus herramientas y bagaje de conocimiento, construir una teoría que permita entender el fenómeno. En ese contexto, conducir una revisión sistemática de fuentes primarias parece una tarea casi imposible (más aún si se considera artículos relativos al tema que no utilicen el término en el título). Afortunadamente, en los últimos dos años, el crecimiento de las publicaciones científicas ha sido acompañado por revisiones sistemáticas de literatura, que facilitan la tarea de aproximación al estado del arte.

Con base en lo expuesto, se ha efectuado una revisión sistemática de revisiones sistemáticas que hayan abordado las noticias falsas en general; en cualquier ámbito o disciplina de la ciencia, a fin de proporcionar un estado del arte de la problemática y su impacto. Para cumplir con el acometido, se planteó como principal hipótesis la existencia de ciertos criterios válidos para medir el impacto de la difusión y consumo de las noticias falsas; así como también las características de los medios que permiten o impiden su difusión. El foco de la revisión se centró en responder a las siguientes preguntas:

- Q1: ¿Qué son las noticias falsas?

- Q2: ¿Qué tipos de noticias falsas existen?

- Q3: ¿Se ha caracterizado el ciclo de vida de las noticias falsas?

- Q4: ¿Qué elementos se utilizan para determinar el impacto de las noticias falsas?

- Q5: ¿Existen estudios que relacionen la propagación o el impacto de las noticias falsas con los medios en que se difunden?

- Q6: ¿Qué estrategias se utilizan para detener o evitar la propagación de las noticias falsas?

Para la revisión sistemática planteada se siguieron los lineamientos metodológicos propuestos por Kitchenham \& Charters (2007). Para facilitar la documentación y trazabilidad de la revisión, se utilizó la plataforma SCOLR (Support for Collaborative, Open, Literature Reviews) del proyecto de investigación CIENTOPOLIS (LIFIA, 2020). Esta plataforma, publicada como portal web, permite efectuar revisiones sistemáticas de literatura, de forma colaborativa, a través de seis etapas: Plan, Carga, Clasificación, Revisión, Reporte, y Discusión.

La búsqueda de los artículos iniciales, efectuada en abril del 2020, se realizó sobre tres repositorios científicos: Scopus, IEEE y ACM. La cadena de búsqueda empleada fue la siguiente:

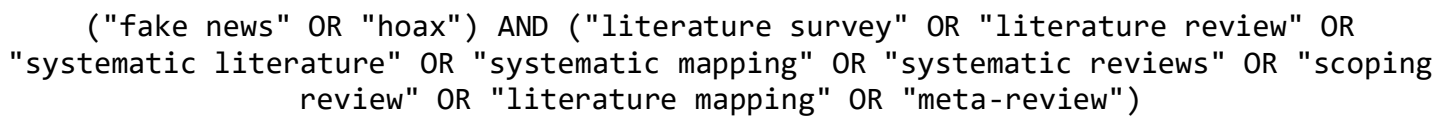

La cadena de búsqueda fue la misma para los tres repositorios; pero con el formato propio de cada uno. Los términos y la combinación de estos fueron buscados en el título, resumen o palabras clave de los artículos, de la siguiente forma:

- Para Scopus: TITLE-ABS-KEY (cadena de búsqueda)

- Para IEEE: "Title": "término" conector "término" / "Abstract": "término" conector "término" o "All Metadata": (cadena de búsqueda)

- Para ACM: Title: (cadena de búsqueda) o Abstract: (cadena de búsqueda) o Keyword: (cadena de búsqueda) 
De la búsqueda en Scopus se obtuvieron 26 artículos. De la búsqueda de ACM se obtuvieron 12 artículos nuevos. La búsqueda en IEEE devolvió 4 artículos, de los cuales 3 ya fueron reportados de las búsquedas en Scopus y ACM; por lo que, se sumó únicamente un artículo nuevo. Al finalizar esta actividad se obtuvieron en total 39 artículos iniciales.

Como siguiente actividad, para la obtención de los artículos que servirían de base para esta investigación, se efectuó una tarea de clasificación. Los criterios empleados fueron:

- Incluir únicamente los artículos que corresponden a revisiones sistemáticas.

- Incluir únicamente los artículos que en su resumen respondan a alguna de las preguntas de investigación planteadas.

- Incluir únicamente artículos en inglés, español y portugués.

Al finalizar el proceso de clasificación se consiguió una base de 14 artículos que fueron revisados de forma pormenorizada.

Tabla 1. Artículos base de la revisión sistemática

\begin{tabular}{|c|c|c|}
\hline Orden & Título & Citación \\
\hline 1 & $\begin{array}{l}\text { The Brazilian Presidential Election of } 2018 \text { and the relationship } \\
\text { between technology and democracy in Latin America. }\end{array}$ & (Tomaz \& Tomaz, 2020) \\
\hline 2 & $\begin{array}{l}\text { Fighting post-truth using natural language processing: A review and } \\
\text { open challenges. }\end{array}$ & (Saquete et al., 2020) \\
\hline 3 & $\begin{array}{l}\text { Fake news detection using machine learning approaches: A systematic } \\
\text { review. }\end{array}$ & (Manzoor et al., 2019) \\
\hline 4 & $\begin{array}{l}\text { The impact of fake news on Social Science Research. Systematized } \\
\text { bibliographic review }\end{array}$ & (Blanco Alfonso et al., 2019) \\
\hline 5 & $\begin{array}{l}\text { Combating Fake News: A Survey on Identification and Mitigation } \\
\text { Techniques. }\end{array}$ & (Sharma et al., 2019) \\
\hline 6 & $\begin{array}{l}\text { Fake news as a two-dimensional phenomenon: a framework and } \\
\text { research agenda. }\end{array}$ & (Egelhofer \& Lecheler, 2019) \\
\hline 7 & $\begin{array}{l}\text { Systematic Literature Review on the Spread of Health-related } \\
\text { Misinformation on Social Media. }\end{array}$ & (Wang et al., 2019) \\
\hline 8 & $\begin{array}{l}\text { The truth (as I see it): philosophical considerations influencing a } \\
\text { typology of fake news. }\end{array}$ & (Ferreira et al., 2019) \\
\hline 9 & $\begin{array}{l}\text { \#Fake news: A systematic review of mechanical thrombectomy results } \\
\text { among neurointerventional stroke surgeons on Twitter. }\end{array}$ & (Dmytriw et al., 2019) \\
\hline 10 & Deepfakes: Threats and countermeasures systematic review. & (Albahar \& Almalki, 2019) \\
\hline 11 & Fake news and social media: The role of the receiver. & (Hooper, 2008) \\
\hline 12 & Fake news: A systematic review of the literature & (Parra \& Oliveira, 2018) \\
\hline 13 & $\begin{array}{l}\text { Epistemology in the Era of Fake News: An Exploration of Information } \\
\text { Verification Behaviors among Social Networking Site Users. }\end{array}$ & (Torres et al., 2018) \\
\hline 14 & Fake News Detection on Social Media: A Data Mining Perspective. & (Shu et al., 2017) \\
\hline
\end{tabular}

\section{Análisis cualitativo}

Los artículos aportan de maneras diversas a los planteamientos realizados acerca de las noticias falsas. Existen revisiones sistemáticas generales y otras que abordan temas específicos de ciertas disciplinas en particular. En cada uno de los puntos que siguen se analizan de manera pormenorizada los aportes.

\subsection{Definiciones de noticias falsas}

El fenómeno de las noticias falsas no es nuevo, la información falsa y distorsionada ha sido característica de la comunicación humana desde la época romana. Sin embargo, en la actualidad y debido a las facilidades que ofrecen los medios tecnológicos, la difusión y el uso de este término se ha vuelto demasiado común. Un aspecto empírico que denota la importancia de las noticias falsas es el aumento exponencial de la producción científica a partir del 2017 (Parra \& Oliveira, 2018). Existen algunas definiciones y significados 
que, dependiendo de la disciplina en la que se estudian o del contexto con el que se describen, permiten comprender el fenómeno y entregan ciertos rasgos o particularidades para identificarlas.

A modo general, el término "noticias falsas" o "fake news" (por su traducción al inglés) se superpone con otros tipos de "información engañosa" como la "información errónea" y la "desinformación", o con cualquier tipo de mensajes u opiniones que se transmiten rápidamente a través de contactos sociales (Wang et al., 2019). En este contexto se aclara que la información errónea es inadvertidamente falsa y que se comparte sin la intención de causar daño alguno; mientras que la desinformación se describe como información fabricada que imita el contenido de los medios de comunicación y que fue creada con la intención de engañar o dañar a una agencia, entidad o persona y/o obtener ganancias financieras o políticas (Hooper, 2008). En este sentido, las noticias falsas se parecen más a la desinformación que a la información errónea.

En una comprensión más amplia, las noticias falsas representan un cambio fundamental en las actitudes políticas y públicas hacia lo que representan el periodismo y las noticias y cómo se pueden obtener los hechos y la información en un mundo digitalizado. La investigación ha comenzado a mostrar que las noticias falsas a menudo se entienden como noticias en las que el lector no cree. El término noticias falsas se emplea sin una definición clara e incluso se utiliza para asociarlo con cualquier declaración que no le gusta o que no es aceptada por el lector.

Existen variadas definiciones, que refuerzan o reprimen ciertas características de la información, dependiendo del ámbito en el que se conciben. El New York Times, por ejemplo, define una noticia falsa como una historia inventada con la intención de engañar y a menudo con motivo de obtener ganancias financieras (Saquete et al., 2020). Las noticias falsas se han tomado también como sinónimo del término post-truth (post-verdad), que según el diccionario de la Oxford se define como "circunstancias en las que hechos objetivos influyen menos en la formación de la opinión pública que lo que lo hacen los llamamientos a emociones y creencias personales". De cierta manera, pueden estar relacionados, puesto que el objetivo de las noticias falsas es influir en la opinión pública y crear inseguridad, sospechas, desestabilización para apoyar o desacreditar o, a veces, simplemente por diversión. Las noticias falsas son un medio para obtener seguidores, perfiles y usuarios de redes sociales con el fin de alentar un movimiento, un interés económico o de marca o a una persona (Parra \& Oliveira, 2018). En un sentido más amplio, y en contraposición de los intereses que las motivan, Sharma et al. (2019) definen a la noticia falsa como un artículo o mensaje noticioso publicado y propagado a través de los medios de comunicación, que contiene información falsa independientemente de los medios y motivos detrás de ella.

De lo recabado en las revisiones sistemáticas, se evidencia que no existe acuerdo en la definición del término noticias falsas; únicamente ciertos criterios de similaridad que nos pueden orientar para la comprensión de una definición personal. Tampoco se ha evidenciado en las revisiones sistemáticas analizadas, una taxonomía referente sobre la que se puedan clasificar los tipos de noticias. No obstante, se mencionan ciertos tipos específicos de noticias falsas que pueden ser incorporados a una posible taxonomía.

Trabajos como el de Egelhofer \& Lecheler (2019) sugieren adoptar un enfoque guiado en la diferencia fundamental de lo que constituye y en lo que se utiliza una noticia falsa: como "género" y como "etiqueta". Se entiende entonces el género como la creación deliberada de desinformación y la etiqueta como la instrumentalización del término para deslegitimar los medios de comunicación. De las noticias falsas como género, se pueden desprender: la propaganda, el rumor y la teoría de conspiración.

Manzoor et al. (2019) referencia la siguiente tipología de noticias falsas:

1. Basadas en lo visual. Utilizan imágenes modificadas o videos manipulados.

2. Basadas en el usuario. Generadas por cuentas falsas y dirigidas a un público específico.

3. Basadas en el conocimiento. Incluyen explicación científica a problemas no resueltos.

4. Basadas en el estilo. Están escritas por pseudoperiodistas con estilo de ciertos periodistas acreditados.

5. Basadas en la postura. Son una representación de declaraciones verídicas que cambian su significado y propósito.

Se definen tipologías más específicas referentes a una disciplina o campo de investigación como en el caso de Ferreira et al. (2019), una tipología de desinformación en relación con marcas (brands). Las autoras definen como atributos de clasificación el "grado de falsedad" y la "fuente de construcción". Con respecto al grado de falsedad se incluyen dos categorías: desinformación fabricada o desinformación basada en hechos veraces; mientras que, con respecto a la fuente de construcción puede ser interna (cuando están 
implicadas) o externa (cuando es víctima). Combinando estos dos atributos se generan los siguientes cuadrantes:

1. The Pinocchio. Totalmente fabricada y construida internamente.

2. White Lie. Basada en hechos falsificados y construida internamente.

3. The Victim. Totalmente fabricada y construida por un actor externo.

4. The Phantom. Enraizada en eventos reales y construida por fuentes externas

Algunos tipos específicos de noticias falsas que se pueden encontrar en los niveles más bajos de la taxonomía pueden ser por ejemplo las deepfake, un tipo de contenido falso que se caracteriza por la manipulación de fotos y videos; especialmente de personajes famosos (Albahar \& Almalki, 2019). Una categorización sensacionalista que puede añadirse también es el clickbait, que se menciona en varios de los artículos revisados. Los titulares de clickbait crean un "vacío de curiosidad", aumentando la probabilidad de que el lector haga clic en el enlace de destino para satisfacer su curiosidad (Shu et al., 2017).

Los tipos de información que pueden excluirse de la categoría de noticias falsas son: la publicidad nativa, debido a que no se crea con la intención de dañar, sino de entretener, y el periodismo pobre puesto que los periodistas pueden introducir información falsa sin intención (Egelhofer \& Lecheler, 2019).

\subsection{Caracterización de las noticias falsas}

Resulta importante conocer las características de las noticias falsas a fin de identificarlas; esto permitirá plantear ciertas medidas que eviten su propagación.

Partiendo de un análisis filosófico, las noticias falsas no pueden ser evaluadas únicamente como una construcción binaria: verdadera o falsa (Ferreira et al., 2019). El "grado de veracidad" es una de las principales características de las noticias falsas. Los niveles de veracidad de las noticias son un tema bastante abordado en las investigaciones e incluso se han categorizado escalas de verdad; un ejemplo de esto es PolitiFact. Torres et al. (2018) exponen que, partiendo de la epistemología del testimonio, la veracidad de la información se puede evaluar bajo cuatro consideraciones: autoridad del testificador; corroboración independiente; plausibilidad y lógica recibida en apoyo; y presentación o medio a través del cual se ofrece el testimonio.

Una característica que está muy relacionada con el grado de veracidad de una noticia es la "credibilidad". Existen tres componentes principales involucrados en la creación, producción, distribución y reproducción de información errónea: la fuente, el medio y el mensaje. Los trabajos de Wang et al. (2019) y Saquete et al. (2020) coinciden en que el concepto de credibilidad abarca: la credibilidad del mensaje, la credibilidad de la fuente y la credibilidad de los medios. La credibilidad de la información en línea puede plantear al lector ciertas inquietudes sobre la confianza con base en esos tres componentes.

Un mensaje o noticia debe estudiarse como noticias falsas cuando tiene poca realidad, se creó con la intención de engañar y se presenta en formato periodístico (Egelhofer \& Lecheler, 2019). Se puede decir que un mensaje tiene poca realidad cuando proviene de una conexión falsa, expone un contexto falso y su contenido es manipulado o engañoso e incluso totalmente falso o contiene elementos deliberadamente engañosos incorporados en su contenido. Las noticias falsas son creadas con la intención de engañar y quienes los producen tienen motivaciones políticas, ideológicas, financieras e incluso la intención de provocar o entretener. Este tipo de mensajes se presentan en formato periodístico; es decir, son una imitación que guarda los componentes estructurales similares a una noticia: poseen un titular, un cuerpo de redacción y, en ocasiones, imágenes. Algunos contenidos falsificados incorporan formatos de noticias de video o de radio. Aunque las noticias falsas se producen con apariencia de información periodística, no se procesan ni se distribuyen por los cauces convencionales del periodismo (Blanco Alfonso et al., 2019). En el trabajo de Manzoor et al. (2019) se indica que existen tres formas principales en las que se publican las noticias: texto, multimedia e hipervínculos. Mientras que el contenido multimedia atrae la atención de los espectadores, los hipervínculos refuerzan su confianza. Algunas páginas que publican este tipo de contenidos crean referencias cruzadas para certificar la génesis de las publicaciones.

La creación de noticias falsas siempre es intencional, mientras que la difusión puede ser involuntaria. La tendencia a difundirse depende del grado en que los lectores sospechen de dicha información falsa (Wang et al., 2019).

Existen otras características que no necesariamente identifican a las noticias falsas, pero que pueden alertar la atención del lector. Egelhofer \& Lecheler (2019) encontraron que las noticias falsas: parecen ser 
más breves y menos informativas que las noticias genuinas; utilizan un lenguaje menos complejo y más personal; es probable que tengan títulos más largos, que contienen el reclamo principal del artículo; y, su contenido está fuertemente influenciado por las agendas de noticias nacionales. Blanco Alfonso et al. (2019) indicaron que el contenido pernicioso de las noticias falsas apela a supuestas noticias llamativas e impactantes; lo cual favorece su propagación. Algunas noticias falsas pueden citar datos verdaderos dentro de un contexto incorrecto para respaldar la afirmación no fáctica (Shu et al., 2017). La temática en la que se generan y difunden contenidos falsos es transversal y abordan todos los campos; por ejemplo: política, medicina, economía, entre otros.

\subsection{Ciclo de vida}

Ninguno de los artículos analizados identifica alguna aproximación referente a un ciclo de vida de las noticias falsas. No se identifican tampoco etapas bien definidas que permitan conocer el grado de evolución de las noticias falsas. Los contenidos revisados ponen en evidencia, implícitamente, que existen posibles etapas como la generación, difusión y aclaración de las noticias falsas. Únicamente en el trabajo de Wang et al. (2019), y a manera general, se definen cuatro etapas marcadas: creación, producción, distribución y reproducción. En la mayoría de los artículos se evidencia que las noticias se generan por diversos tipos de motivaciones o intereses; es decir, concuerdan con la etapa de creación. La etapa de producción, en cambio, no está bien delimitada; por lo que, puede confundirse con la creación o con los procedimientos de generación de las noticias falsas: como la edición de contenidos en portales web o la elaboración de deepfake (Albahar \& Almalki, 2019). La etapa de distribución se la puede asociar con la difusión de los contenidos. Y, por último, la etapa de reproducción resulta también ambigua, debido a que se puede confundir con el consumo de la información o con su reenvío.

\subsection{Formas de creación y propagación}

Para analizar las causas que originan las noticias falsas se puede partir de los intereses que impulsan la generación de estos contenidos. Según Saquete et al. (2020), existen dos tipos de intereses principales que las motivan: los ideológicos, que apuntan a manipular la opinión social para enfocar a las personas en pensar o actuar de una manera específica; y los económicos, que impulsan a los individuos o las empresas a ganar dinero mediante clickbaits. Shu et al. (2017) reafirman que las noticias falsas producidas en línea tienen propósitos financieros y de ganancia política.

Relacionado a los intereses políticos, la Web representa un canal de comunicación directo entre el elector y el poder político; una vez dentro de la web todos somos comunicadores. En este sentido, un factor que facilita la difusión de las noticias falsas es la denominada calificación cero, que provoca cierto colonialismo digital que vincula a los usuarios con el uso de ciertas aplicaciones y servicios; por ejemplo, el WhatsApp en Brasil (Tomaz \& Tomaz, 2020). Los planes de internet con calificación cero son aquellos que ofrecen un subsidio a redes sociales y servicios de mensajería, entre los principales están Facebook, Twitter y WhatsApp; condicionando al lector a consumir lo que se comparte en esos medios e impidiendo el acceso a portales más serios o verificados.

En cuanto a los intereses económicos, el público puede participar en el proceso de producción y difusión. La publicidad digital permite que las noticias falsas sean financieramente atractivas, ya que las visitas o los "clics" en lugar de la precisión del contenido crean éxito comercial (Egelhofer \& Lecheler, 2019). Se han desarrollado modelos de negocio que se pueden clasificar como: generadores, clonadores, pensadores ilusorios, ciudadanos satíricos y facilitadores (Hooper, 2008). Se diferencian incluso dos categorías: el desarrollador de noticias falsas y el verificador de noticias falsas.

De acuerdo con lo evidenciado en las revisiones sistemáticas analizadas, las noticias falsas se difunden principalmente por: medios tradicionales como diarios, radio y televisión (Shu et al., 2017); y por redes sociales y páginas especializadas en sátira. Los espacios colaborativos más mencionados han sido Facebook, WhatsApp y Twitter. El éxito en la difusión de una noticia falsa tiene que ver muchas veces con que la verificación de hechos se comparte con un retraso significativo después de la difusión de la información falsa original (Egelhofer \& Lecheler, 2019). En ocasiones, aun cuando los medios tradicionales o los sitios especializados de noticias validan un hecho de manera oportuna, el exceso y la repetición tienen más efecto que una secuencia lógica de los argumentos presentados para validarlo (Tomaz \& Tomaz, 2020). Una vez que se forma la percepción errónea, es muy difícil corregirla: la corrección de información falsa, 
mediante la presentación de información fáctica, es inútil e incluso puede aumentar las percepciones erróneas, especialmente entre grupos ideológicos (Shu et al., 2017).

La información errónea es la más popular entre los usuarios y gran parte de ella proviene de personas que son muy activas para influir en las opiniones. En las elecciones presidenciales estadounidenses de 2016, por ejemplo, las noticias falsas más populares se difundieron aún más que las noticias auténticas (Shu et al., 2017). En política, los discursos de odio promueven la difusión de noticias falsas (Tomaz \& Tomaz, 2020). En salud "los lazos fuertes entre la comunidad son obstáculos para que se acepten las opiniones de expertos" (Wang et al., 2019). Los usuarios epistemológicamente ingenuos tienen una mayor propensión a compartir rumores de salud en línea.

Para comprender el fenómeno de difusión de las noticias falsas, según Wang et al. (2019), se emplean estudios de teorías de sistemas o redes que explican los patrones de influencia, aprendizaje y contagio social; además de los procesos de homofilia y polarización. En el mismo trabajo se referencia investigación existente acerca de la dinámica de la información falsa, mediante el estudio de las redes sociales y los modelos epidemiológicos. La técnica recurrente para investigar la viralización de las noticias falsas es el estudio del Social Network Analysis (Blanco Alfonso et al., 2019).

\subsection{Características de usuarios y medios de difusión}

La difusión de las noticias falsas depende en gran medida del criterio y la acción de los lectores. La cantidad de rumor en circulación dependerá de la importancia del tema para los individuos y en ocasiones de la ambigüedad de la evidencia (Wang et al., 2019). En el fenómeno de la difusión los usuarios son autoeditores, sin ninguna responsabilidad o forma de verificar los hechos que publican. Por lo general, los usuarios difunden información falsa si es que los contenidos le inducen emociones negativas como el miedo, la ira o la tristeza. Según Manzoor et al. (2019), existen factores psicológicos que convencen a los lectores a creer en noticias falsas. Los lectores poseen cierto tipo de fascinación con lo impactante, de modo que no importa si la información es falsa, igual la compartirán para deleitarse con los detalles (Hooper, 2008). Los agentes que generan información falsa son en su mayoría individuos sin filiaciones oficiales o institucionales.

En la revisión realizada por Shu et al., (2017), se expone la existencia de dos principios que hacen que los consumidores sean naturalmente vulnerables: el realismo ingenuo que provoca que los consumidores tiendan a creer que sus percepciones de la realidad son las únicas opiniones precisas, mientras que los otros, que no están de acuerdo, sean considerados como desinformados, irracionales o sesgados; y el sesgo de confirmación que infiere en que los consumidores prefieren recibir información que confirme sus opiniones. Estos principios son citados también por Sharma et al. (2019) y además se suma un tercero: la teoría de la influencia normativa que sugiere que los individuos eligen compartir y consumir opciones socialmente seguras como preferencia por la aceptación y afirmación social. Esto implica que los individuos busquen, consuman y compartan información que esté alineada con sus propios puntos de vista e ideologías.

Las redes sociales grandes y diversificadas permiten un crecimiento exponencial de las noticias (Manzoor et al., 2019). Facebook, por ejemplo, mejora el intercambio y "me gusta" de las publicaciones, lo que facilita la difusión pública de las noticias falsas. En WhatsApp, se reduce la posible vergüenza de compartir información falsa debido a su naturaleza privada (Tomaz \& Tomaz, 2020). Twitter es particularmente adecuada para la difusión de noticias falsas, debido a que la limitación a 140 caracteres permite al remitente enviar la información suficiente para estimular el interés sin demasiada información cuestionable (Hooper, 2008). Si bien los medios juegan un papel importante en la difusión de contenidos, es el usuario quien define el éxito o fracaso de la comunicación.

\subsection{Estrategias para detener o evitar la propagación}

La literatura disponible enfatiza en los múltiples esfuerzos por evitar la propagación de las noticias falsas. Existen muchas técnicas de detección automática; varias de las cuales utilizan inteligencia artificial, especialmente ciertos métodos del aprendizaje automático (Manzoor et al., 2019). Sin embargo, aún no se evidencian métodos de alta confiabilidad que garanticen el consumo de información verídica.

Gran parte de los artículos analizados coinciden en que la mejor estrategia para evitar el consumo y difusión de noticias falsas es la combinación de la detección asistida por computadora con la alfabetización en información y la promoción del pensamiento crítico del ciudadano. Una medida acertada para educar a 
los individuos es darles a conocer las infografías de identificación de las noticias falsas (Parra \& Oliveira, 2018). Tomaz \& Tomaz (2020) mencionan que organizaciones como O Truco y Aos Fatos, han trabajado en la traducción de guías, producidas por la International Fact-Checking Network (IFCN), para verificación de noticias y lucha contra las noticias falsas. Es fundamental equipar a la comunidad para que sean capaces de identificar noticias a través de la validación (Torres et al., 2018). Mientras más realistas parecen ser mayor es el impacto en los lectores y más complicada su identificación. El proceso de verificación de los hechos debe ser transparente y las plataformas de intercambio de noticias deben ofrecer un entorno neutral para el debate. Exponer a las personas a medidas de opinión colectiva podría reducir la tendencia a compartir información errónea (Wang et al., 2019). Parra \& Oliveira (2018) referencian que las propuestas concretas se están implementando en torno a tres medios: 1. Reclutamiento de revisores o editores procedentes de medios de comunicación; 2. Estrategia colaborativa (crowdsourcing); y 3. Soluciones tecnológicas basadas en algoritmos.

Para la construcción de modelos de detección automática se trabaja en función de la meta información de la noticia: la fuente, el título, el texto del mensaje y la imagen o video incluidos (Shu et al., 2017). Aprovechando esta información se pueden extraer características de contenido y de contexto social, que luego forman parte de un corpus que puede ser analizado utilizando técnicas de minería de datos. Adicional a lo indicado, es también importante analizar las respuestas de los usuarios; principalmente porque estas, junto con los patrones de propagación, son más difíciles de manipular que el contenido del mensaje y, a menudo, contienen información obvia sobre la veracidad (Sharma et al., 2019).

Las noticias falsas han ganado tanta notoriedad que las empresas tecnológicas están trabajando en reducir el impacto en sus plataformas (Torres et al., 2018). Facebook, por ejemplo, está ofreciendo formas para que los usuarios las marquen. Google está integrando la verificación de datos en Google Search y Google News para minimizar la difusión de contenidos falsos. Y, Wikipedia se encuentra desarrollando Wikitribune un sitio que ocupa crowdsourcing para verificar las fuentes de noticias.

\subsection{Impacto de las noticias falsas}

Uno de los puntos de análisis que mayor importancia cobra en el estudio de las noticias falsas, es el impacto que éstas producen en individuos, entidades y sociedades. La propagación de información intencionalmente engañosa puede alterar las interacciones sociales y dañar vidas individuales (Shu et al., 2017). Las consecuencias son múltiples y los ejemplos de situaciones trágicas o críticas son incontables. En la revisión sistemática efectuada no se ha encontrado ninguna metodología para determinar el impacto que producen las noticias falsas. Ninguno de los artículos cuantifica el daño ocasionado a nivel de integridad de las personas, entidades o sociedades; únicamente se exponen ciertos ejemplos que facilitan una cierta comprensión.

En el ámbito de la política, las noticias falsas han tenido influencia en los procesos electorales de ciertos países (Parra \& Oliveira, 2018; Tomaz \& Tomaz, 2020). Afectan la confianza y dan forma a la precepción que una persona tiene sobre los demás; pudiendo influir en los debates políticos y en las opiniones de las noticias serias (Torres et al., 2018). La estabilidad de muchos gobiernos depende en gran medida de cómo manejan para sí las publicaciones de contenidos falsos. Algunos actores políticos usan las noticias falsas para desacreditar a los medios de comunicación que contradicen sus posiciones (Egelhofer \& Lecheler, 2019).

En el ámbito financiero el impacto que ocasiona la desinformación se puede medir en términos económicos y de reputación (Ferreira et al., 2019).

Dmytriw et al. (2019) dan a entender que, cuando se trata de temas de salud, aparte de las noticias falsas, existe el riesgo asociado al sesgo de las publicaciones médicas que se realizan en redes sociales como Twitter, donde los profesionales publican únicamente los resultados satisfactorios. Las consecuencias pueden ser fatales debido a las expectativas poco realistas de terapias milagrosas.

\subsection{Relación entre la propagación e impacto y los espacios colaborativos}

Uno de los cuestionamientos de esta investigación fue conocer la relación que existe entre la propagación y el impacto de las noticias falsas y los medios en los que se difunden. En este sentido se ha prestado especial atención a los medios referenciados en los artículos analizados y las características que facilitan o impiden la propagación de las noticias falsas. Sin embargo, no se ha encontrado evidencia relevante al 
respecto. Aunque se mencionan espacios colaborativos como WhatsApp, Facebook o Twitter, ningún artículo profundiza en las características de los medios ni en su influencia en la propagación o en el impacto de las noticias falsas. Wang et al. (2019) alcanzan a referenciar que Ozturk et al. (2015) explora cómo las diferentes configuraciones de las redes sociales pueden reducir la propagación de los rumores basándose en la investigación de la psicología de los rumores.

\section{Conclusiones}

El fenómeno de las noticias falsas resulta bastante complejo. La visión global que se brinda ha permitido identificar ciertos aspectos fundamentales de la caracterización de estos contenidos como: las motivaciones por las que se producen; las formas y formatos en los que se crean y el "grado de veracidad" de la información que contienen. Como punto de partida se ha conseguido diferenciar el término "noticias falsas" de otros como "desinformación" y "post-verdad". Se deja por sentado que un contenido debe analizarse como noticia falsa, únicamente cuando tiene poca realidad, fue creado con la intención de engañar y se presenta en formato periodístico. Los artículos analizados han respondido, en mayor o menor grado, a todas las interrogantes planteadas al inicio de la investigación. Los hallazgos documentados han permitido proponer un panorama completo de las noticias falsas: aclarando las definiciones; diferenciando los tipos de noticias; revelando las características de los usuarios y medios, que facilitan o impiden su difusión; analizando el impacto que producen; y exponiendo los aspectos que no han sido investigados. Finalmente se puede comprender que la educación del lector es un aspecto importante para evitar la propagación de las noticias falsas y que la mejor estrategia propuesta para frenar su difusión es la combinación de las técnicas asistidas por computadora, la alfabetización en información y la promoción del pensamiento crítico del ciudadano. Un aspecto importante que merece especial atención es la falta de evidencia de estudios relacionados con el impacto de las noticias falsas. No se evidencian propuestas metodológicas para evaluar el impacto; ya sea de manera cuantitativa o cualitativa. Y, por último, se cuenta como un buen hallazgo de la relación existente entre las características de los espacios colaborativos y la difusión de las noticias falsas.

\section{Declaración de conflicto de intereses}

Los autores declaran no tener conflicto de intereses con respecto a la investigación, autoría o publicación de este artículo.

\section{Referencias}

Albahar, M., \& Almalki, J. (2019). Deepfakes: Threats And Countermeasures Systematic Review. Journal of Theoretical and Applied Information Technology, 97(22).

Blanco Alfonso, I., García Galera, C., \& Tejedor Calvo, S. (2019). The impact of fake news on social science research. Systematized bibliographical review. Historia y Comunicación Social, 24(2), 449-469. https://doi.org/10.5209/hics.66290

Dmytriw, A. A., Sorenson, T. J., Morris, J. M., Nicholson, P. J., Hilditch, C. A., Graffeo, C. S., \& Brinjikji, W. (2019). \#Fake news: a systematic review of mechanical thrombectomy results among neurointerventional stroke surgeons on Twitter. Journal of NeuroInterventional Surgery, 11(5), 460-463. https://doi.org/10.1136/neurintsurg-2018-014319

Egelhofer, J. L., \& Lecheler, S. (2019). Fake news as a two-dimensional phenomenon: a framework and research agenda. Annals of the International Communication Association, 43(2), 97-116. https://doi.org/10.1080/23808985.2019.1602782

Ferreira, C. C., Robertson, J., \& Kirsten, M. (2019). The truth (as I see it): philosophical considerations influencing a typology of fake news. Journal of Product \& Brand Management, 29(2), 150-158. https://doi.org/10.1108/JPBM-12-2018-2149

Hooper, V. A. (2008). Fake news and social media: The role of the receiver. En N. Corcoran \& V. Cunnane (Eds.), Proceedings of the 5th European Conference on Social Media, ECSM 2018 (pp. 62-68). Academic Conferences and Publishing International Limited.

Kitchenham, B., \& Charters, S. (2007). Guidelines for performing Systematic Literature Reviews in Software Engineering. En Keele University and Durham University Joint Report. 
LIFIA. (2020). Scolr. Support for Collaborative, Open, Literature Reviews. http://scolr.cientopolis.org

Manzoor, S. I., Singla, J., \& Nikita. (2019). Fake News Detection Using Machine Learning approaches: A systematic Review. 2019 3rd International Conference on Trends in Electronics and Informatics (ICOEI), 230-234. https://doi.org/10.1109/ICOEI.2019.8862770

Ozturk, P., Li, H., \& Sakamoto, Y. (2015). Combating Rumor Spread on Social Media: The Effectiveness of Refutation and Warning. 2015 48th Hawaii International Conference on System Sciences, 2406-2414. https://doi.org/10.1109/HICSS.2015.288

Parra, P., \& Oliveira, L. (2018). Fake news: A systematic review of the literature. Observatorio, 12, 54-78.

Saquete, E., Tomás, D., Moreda, P., Martínez-Barco, P., \& Palomar, M. (2020). Fighting post-truth using natural language processing: A review and open challenges. En Expert Systems with Applications (Vol. 141, p. 112943). Elsevier Ltd. https://doi.org/10.1016/j.eswa.2019.112943

Sharma, K., Qian, F., Jiang, H., Ruchansky, N., Zhang, M., \& Liu, Y. (2019). Combating Fake News: A Survey on Identification and Mitigation Techniques. ACM Transactions on Intelligent Systems and Technology, 10, 1-42. https://doi.org/10.1145/3305260

Shu, K., Sliva, A., Wang, S., Tang, J., \& Liu, H. (2017). Fake News Detection on Social Media: A Data Mining Perspective. ACM SIGKDD Explorations Newsletter, 19(1), 22-36. https://doi.org/10.1145/3137597.3137600

Tomaz, R. M., \& Tomaz, J. M. T. (2020). The Brazilian Presidential Election of 2018 and the relationship between technology and democracy in Latin America. Journal of Information, Communication and Ethics in Society, 18(4), 497-509. https://doi.org/10.1108/JICES-12-2019-0134

Torres, R., Gerhart, N., \& Negahban, A. (2018). Epistemology in the Era of Fake News: An Exploration of Information Verification Behaviors among Social Networking Site Users. ACM SIGMIS Database: the DATABASE for Advances in Information Systems, 49(3), 78-97. https://doi.org/10.1145/3242734.3242740

Wang, Y., McKee, M., Torbica, A., \& Stuckler, D. (2019). Systematic Literature Review on the Spread of Healthrelated Misinformation on Social Media. Social Science \& Medicine, 240, 112552. https://doi.org/10.1016/j.socscimed.2019.112552 